\title{
Quantum corrections to the mass of the supersymmetric vortex
}

\author{
D. V. Vassilevich* \\ Institut für Theoretische Physik, Univeristät Leipzig, \\ Augustusplatz 10, 04109 Leipzig, Germany
}

November 10, 2018

\begin{abstract}
We calculate quantum corrections to the mass of the vortex in $N=2$ supersymmetric abelian Higgs model in $2+1$ dimensions. We put the system in a box and apply the zeta function regularization. The boundary conditions inevitably violate a part of the supersymmetries. Remaining supersymmetry is however enough to ensure isospectrality of relevant operators in bosonic and fermionic sectors. A non-zero correction to the mass of the vortex comes from finite renormalization of couplings.
\end{abstract}

PACS: 11.27.+d; 12.60.Jv

\section{Introduction}

The Abrikosov-Nielsen-Olesen (ANO) vortices [1] play an important role in modern particle physics [2]. In particular, supersymmetric ANO vortices are essential for understanding of the monopole condensation (see, e.g., [3] and references therein). In $2+1$ dimensions the relation between extended $N=2$ supersymmetry and the BPS bound has been demonstrated in [4 following a more general discussion of [5].

Quantum corrections to the mass of the supersymmetric ANO vortex ${ }^{1}$ in $2+1$ dimensions were calculated in [7], the Chern-Simons terms were included in [8]. Both papers [7,8] give zero result for the mass shift. The authors used

*Also at V. A. Fock Institute of Physics, St. Petersburg University, 198904 St. Petersburg, Russia; e.mail: Dmitri.Vassilevich@itp.uni-leipzig.de

${ }^{1}$ Since no analytic form for the profile functions of the ANO vortex is available, calculations of the mass shift in a non-supersymmetric case is a rather complicated problem. Recently the fermionic contribution to the vacuum energy was calculated in a toy model closely resembling the abelian Higgs model $[\underline{6}$. 
the arguments similar to that of Imbimbo and Mukhi $[9]^{2}$ based on the nonlocal index theorem of [10] and its generalisation by E. Weinberg [11]. Roughly speaking, the line of reasoning in [7,8] was as follows. The index theorem was used to show that

$$
\rho_{B}(\omega)-\rho_{F}(\omega) \propto \delta(\omega),
$$

where $\rho_{B, F}$ are the spectral densities in the bosonic and fermionic sectors respectively. Then the mass shift was identified as

$$
\Delta E \propto \int d \omega \omega\left(\rho_{B}(\omega)-\rho_{F}(\omega)\right)
$$

Due to (11) the mass shift (2) should be zero. In this way, the authors [7,8, avoided explicit use of any regularization. There is however a loophole in this kind of arguments. First of all, it is assumed that there is a regularization which supports the mode-by-mode cancellations needed to apply (2). Such regularizations are indeed available. One of them is the zeta function regularization 12. However, it requires transition to the discrete spectrum at least at intermediate steps. In other words, one has to put the system in a box and impose some boundary conditions. There is no a priori guarantee that these boundary conditions can be chosen in such a way to preserve the index theorem arguments. Besides, without regularising the whole theory with arbitrary, not only BPS background fields, one cannot control finite renormalization of charges which are present in the model.

Quantum corrections to $2+1$ dimensional solitons should have been reconsidered already some time ago. Recent years have seen a considerable increase of interest to quantum effects around supersymmetric solitons in $1+1$ dimensions initiated by the papers [13] resulted in some very interesting developments in this field (see [14] for a literature survey).

In this paper we re-calculate quantum corrections to the mass of the supersymmetric vortex using the method [15] applied previously to the supersymmetric kink. We put the vortex in a box with a circular boundary and impose the boundary condition which preserve as many symmetries as possible. We find that one half of the supersymmetries of the vortex is inevitably broken at the bound$\operatorname{ary}^{3}$. This is however, enough to ensure coincidence of the eigenfrequencies of the bosonic and fermionic fluctuations. We then conclude that the total energy of the vortex and the boundaries is zero. At the next step, we define the energy associated with the boundaries and find that it is also zero. Therefore, the whole mass shift of the vortex is due to the finite renormalization of couplings ${ }^{4}$. It is

\footnotetext{
${ }^{2}$ Note, that the authors 9 used these arguments to show saturation of the Bogomolny bound (i.e. to estimate the difference between quantum corrections to the mass and to the central charge) rather than to calculate the mass shift itself.

${ }^{3}$ BPS states preserve a half of the supersymmetries of the theory. Since boundaries break another half, we have a quarter of the original $N=2$ supersymmetry.

${ }^{4} \mathrm{I}$ am gratefull to R. Wimmer for pointing out imporatnce of the finite renormalization effects.
} 
not zero and is given by the equation (170) below.

Formally the zero point energy can be represented as

$$
\Delta E=\Delta E_{B}-\Delta E_{F}, \quad \Delta E_{B, F}=\frac{1}{2} \sum_{\omega_{B, F}} \omega_{B, F},
$$

where $\omega_{B, F}$ are eigenfrequencies of bosonic and fermionic fluctuations. The sums in (31) are divergent and must be regularized. We use the zeta function regularization [12]:

$$
\Delta E_{B, F}^{\mathrm{reg}}=\frac{1}{2} \sum_{\omega_{B, F} \neq 0} \omega_{B, F}^{1-2 s},
$$

where $s$ is the regularization parameter. Note, that zero frequencies (which do not contribute to (3) anyhow) should be explicitly excluded.

This paper is organised as follows. In the next section we describe properties of classical solutions and define the operators acting on quantum fluctuation in the abelian Higgs model without boundaries. In sec. 3] we define gauge invariant boundary conditions which ensure that all non-zero eigenfrequencies in fermionic and bosonic sectors coincide. In sec. 4 we analyse supersymmetry of these boundary conditions and find that one half of the superinvariancies of the vortex is broken. Section 5 is devoted to calculation of the mass shift. Some concluding remarks are given in sec. 6. Technical details of the calculations related to the boundary supersymmetries are presented in the Appendix.

\section{The model}

This section is devoted to some known properties of the supersymmetric vortices on manifolds without boundaries. Here we mostly follow [16, 8.

\subsection{Classical theory}

The Lagrangian of $N=2$ supersymmetric abelian Higgs model in $(2+1)$ dimensions reads:

$$
\begin{aligned}
& \mathcal{L}=\mathcal{L}_{B}+\mathcal{L}_{F} \\
& \mathcal{L}_{B}=-\frac{1}{4} F_{\mu \nu} F^{\mu \nu}-\left|D_{\mu} \phi\right|^{2}-\frac{1}{2}\left(\partial_{\mu} w\right)^{2}-\frac{e^{2}}{2}\left(|\phi|^{2}-v^{2}\right)^{2}-e^{2} w^{2}|\phi|^{2}, \\
& \mathcal{L}_{F}=i \bar{\psi} \gamma^{\mu} D_{\mu} \psi+i \bar{\chi} \gamma^{\mu} \partial_{\mu} \chi-i \sqrt{2} e\left(\bar{\psi} \chi \phi-\bar{\chi} \psi \phi^{*}\right)+e w \bar{\psi} \psi
\end{aligned}
$$

where $w$ (respectively, $\phi$ ) is real (respectively, complex) scalar, $\psi$ and $\chi$ are twocomponent complex spinors. $v$ is a constant. The signature of the metric $g^{\mu \nu}$ is $(-++)$. As usual, $F_{\mu \nu}=\partial_{\mu} A_{\nu}-\partial_{\nu} A_{\mu}$ is the field strength. $D_{\mu}$ is gauge 
covariant derivative, $D_{\mu} \phi=\left(\partial_{\mu}-i e A_{\mu}\right) \phi$. The action (5) is invariant under the supersymmetry transformations

$$
\begin{aligned}
& \delta A_{\mu}=i\left(\bar{\eta} \gamma_{\mu} \chi-\bar{\chi} \gamma_{\mu} \eta\right) \\
& \delta \phi=\sqrt{2} \bar{\eta} \psi, \quad \delta w=i(\bar{\chi} \eta-\bar{\eta} \chi) \\
& \delta \chi=\gamma^{\mu} \eta\left(\partial_{\mu} w+\frac{i}{2} \epsilon_{\mu \nu \lambda} F^{\nu \lambda}\right)+i \eta\left(e|\phi|^{2}-e v^{2}\right) \\
& \delta \psi=-\sqrt{2}\left(i \gamma^{\mu} \eta D_{\mu} \phi-\eta e w \phi\right)
\end{aligned}
$$

with complex constant spinor parameter $\eta$. $\epsilon^{\mu \nu \rho}$ is the Levi-Civita tensor, $\epsilon^{012}=1$. The gamma matrices

$$
\gamma^{0}=\left(\begin{array}{cc}
1 & 0 \\
0 & -1
\end{array}\right), \quad \gamma^{1}=\left(\begin{array}{cc}
0 & 1 \\
-1 & 0
\end{array}\right), \quad \gamma^{2}=\left(\begin{array}{cc}
0 & i \\
i & 0
\end{array}\right)
$$

satisfy the equation

$$
\gamma^{\mu} \gamma^{\nu}=-g^{\mu \nu}-i \epsilon^{\mu \nu \rho} \gamma_{\rho}
$$

We shall mark upper (lower) components of all spinors with the subscript "+" (respectively, "-"), so that

$$
\eta=\left(\begin{array}{c}
\eta_{+} \\
\eta_{-}
\end{array}\right)
$$

for example.

Consider now static bosonic field configurations such that $A_{0}=w=0$. Such configurations are invariant with respect to one half of the supersymmetry transformations (8) corresponding to $\eta_{+}=0$ if and only if

$$
\begin{aligned}
& \left(D_{1}+i D_{2}\right) \phi=0, \\
& F_{12}+e\left(|\phi|^{2}-v^{2}\right)=0 .
\end{aligned}
$$

These are just the Bogomolny [17] self-duality equations.

The classical vortices

$$
\phi=f(r) e^{i n \theta}, \quad e A_{j}=\epsilon_{j k} \frac{x^{k}}{r^{2}}(a(r)-n)
$$

satisfy (12) and (13) if

$$
\begin{aligned}
& \frac{1}{r} \frac{d}{d r} a(r)=e^{2}\left(f^{2}(r)-v^{2}\right), \\
& r \frac{d}{d r} \ln f(r)=a(r) .
\end{aligned}
$$

In these equations $n \in \mathbb{N}$ is vorticity (which we assume to be positive), $j, k \in$ $\{1,2\}, \epsilon_{12}=1$, and $r, \theta$ are usual polar coordinates on the plane. The functions $f(r)$ and $a(r)$ satisfy the conditions

$$
\begin{array}{ll}
f(0)=0, & f(\infty)=v, \\
a(0)=n, & a(\infty)=0 .
\end{array}
$$


The classical energy of this configuration reads (see, e.g. [8]):

$$
E^{\mathrm{cl}}=2 \pi n v^{2} .
$$

\subsection{Quantum fluctuations}

Let us now turn to quantum fluctuations about the background (14). We shift $\phi \rightarrow \phi+\varphi$ and $A_{\mu} \rightarrow A_{\mu}+\alpha_{\mu}$, where $\varphi$ and $\alpha_{\mu}$ are the fluctuations. Since all other fields are zero on the background, we do not need to introduce more notations. It is convenient to use the background gauge fixing term ${ }^{5}$

$$
\mathcal{L}_{\mathrm{gf}}=-\frac{1}{2}\left[\partial_{\mu} \alpha^{\mu}-i e\left(\varphi^{*} \phi-\varphi \phi^{*}\right)\right]^{2},
$$

which generates the following action for the complex ghosts $\sigma$ :

$$
\mathcal{L}_{\text {ghost }}=\sigma^{*}\left(\partial_{\mu} \partial^{\mu}-2 e^{2} \phi \phi^{*}\right) \sigma .
$$

Next we expand the action (6) about the classical background. The terms linear in fluctuations vanish due to the equations of motion. In the next, quadratic, order we have in the bosonic sector:

$$
\begin{gathered}
\mathcal{L}_{B}^{2}+\mathcal{L}_{\mathrm{gf}}=-\frac{1}{2} \alpha_{\mu}\left(\square-2 e^{2} \phi^{*} \phi\right) \alpha^{\mu}-\left(D_{\mu} \varphi\right)\left(D^{\mu} \varphi\right)^{*}-e^{2} \varphi \varphi^{*}\left(3 \phi \phi^{*}-v^{2}\right) \\
-2 i e \alpha^{\mu}\left(\varphi^{*} D_{\mu} \phi-\varphi D_{\mu} \phi^{*}\right)-\frac{1}{2}\left(\partial_{\mu} w\right)^{2}-e^{2} w^{2}|\phi|^{2},
\end{gathered}
$$

where the covariant derivative $D_{\mu}$ depends on on the background gauge potential $A_{\mu} ; \square=\partial_{\mu} \partial^{\mu}$.

The quadratic part of the fermionic action coincides with (17) where all bosonic fields take their background values (so that $w=0$, for example). Therefore, the equation which defines eigenfrequencies $\omega_{F}$ in the fermionic sector reads:

$$
\omega_{F}\left(\begin{array}{l}
\psi \\
\chi
\end{array}\right):=i \partial_{0}\left(\begin{array}{l}
\psi \\
\chi
\end{array}\right)=-i \gamma^{0}\left(\begin{array}{cc}
\gamma^{j} D_{j}, & -\sqrt{2} e \phi \\
\sqrt{2} e \phi^{*}, & \gamma^{k} \partial_{k}
\end{array}\right)\left(\begin{array}{l}
\psi \\
\chi
\end{array}\right) .
$$

By taking square of this equation one obtains [8]:

$$
\omega_{F}^{2}\left(\begin{array}{c}
U \\
V
\end{array}\right)=\left(\begin{array}{cc}
D_{F} D_{F}^{\dagger}, & 0 \\
0, & D_{F}^{\dagger} D_{F}
\end{array}\right)\left(\begin{array}{c}
U \\
V
\end{array}\right)
$$

where

$$
U=\left(\begin{array}{c}
\psi_{+} \\
\chi_{-}
\end{array}\right), \quad V=\left(\begin{array}{c}
\psi_{-} \\
\chi_{+}
\end{array}\right)
$$

\footnotetext{
${ }^{5}$ This gauge condition belongs to the so-called $R_{\xi}$ family [18].
} 
and

$$
D_{F}=\left(\begin{array}{cc}
D_{+}, & -\sqrt{2} e \phi \\
-\sqrt{2} e \phi^{*}, & \partial_{-}
\end{array}\right), \quad-D_{F}^{\dagger}=\left(\begin{array}{cc}
D_{-}, & \sqrt{2} e \phi \\
\sqrt{2} e \phi^{*}, & \partial_{+}
\end{array}\right) .
$$

In this equation we have used holomorphic and anti-holomorphic components of two-dimensional differential operators:

$$
D_{ \pm}:=D_{1} \pm i D_{2}, \quad \partial_{ \pm}:=\partial_{1} \pm i \partial_{2} .
$$

Let us now return to the bosonic fluctuation. As one can see from (21), the equations for $\alpha_{0}$ and for $w$ decouple from the rest of the bosonic modes. Moreover, the squared eigenfrequencies of these fields are given by the eigenvalues of the operator

$$
\Delta_{w}=-\partial_{j} \partial_{j}+2 e^{2}|\phi|^{2} .
$$

The same operator defines also the ghost eigenfrequencies. Therefore, contributions of all these fields to the vacuum energy cancel (provided they all satisfy the same boundary conditions).

A very important observation regarding the rest of the bosonic perturbations was made by Lee and Min [8]. They demonstrated that the eigenfrequencies for $\varphi$ and $\alpha_{j}$ follow from the equation:

$$
\omega_{B}^{2}\left(\begin{array}{c}
\varphi \\
i \alpha_{+} \sqrt{2}
\end{array}\right):=-\partial_{0}^{2}\left(\begin{array}{c}
\varphi \\
i \alpha_{+} \sqrt{2}
\end{array}\right)=D_{F}^{\dagger} D\left(\begin{array}{c}
\varphi \\
i \alpha_{+} \sqrt{2}
\end{array}\right),
$$

where $\alpha_{+}=\alpha_{1}+i \alpha_{2}$. One can check this statement by a direct calculation using the Bogomolny equations (12) and (13) for the background fields.

For the sake of completeness we give here explicit expressions for $D_{F} D_{F}^{\dagger}$ and $D_{F}^{\dagger} D_{F}$ :

$$
\begin{aligned}
& D_{F}^{\dagger} D_{F}=-\left(\begin{array}{cc}
D_{j}^{2}-e^{2}\left(3|\phi|^{2}-v^{2}\right), & -\sqrt{2} e\left(D_{-} \phi\right) \\
-\sqrt{2} e\left(D_{+} \phi^{*}\right), & \partial_{j}^{2}-2 e^{2}|\phi|^{2}
\end{array}\right), \\
& D_{F} D_{F}^{\dagger}=-\left(\begin{array}{cc}
D_{j}^{2}-e^{2}\left(|\phi|^{2}+v^{2}\right), & 0 \\
0, & \partial_{j}^{2}-2 e^{2}|\phi|^{2}
\end{array}\right) .
\end{aligned}
$$

We stress that these formulae are valid only if the background satisfies the Bogomolny equations.

\section{Boundary conditions}

The aim of this section is to define the boundary conditions which support the factorisation properties of the eigenfrequency equations (23) and (28). We like to keep as much symmetry between the bosonic and fermionic fluctuation as possible. 
Let us put the system in a spherical shell with the boundary at $r=R$ (the time coordinate $x^{0}$ remains, of course, unrestricted). The relation

$$
u_{ \pm}=e^{ \pm i \theta}\left(u_{r} \pm \frac{i}{r} u_{\theta}\right)
$$

between complex and angular representations of an arbitrary two-vector $u_{j}$ will be useful in this and subsequent sections.

We start with gauge invariant boundary conditions for $\alpha_{\mu}$ and $\sigma$. By gauge invariance we understand the following property [19]. Let $\mathcal{B}^{[\alpha]}$ and $\mathcal{B}^{[\sigma]}$ be boundary operators which define boundary conditions for $\alpha$ and $\sigma$ respectively:

$$
\left.\mathcal{B}^{[\alpha]} \alpha_{\mu}\right|_{\partial M}=0,\left.\quad \mathcal{B}^{[\sigma]} \sigma\right|_{\partial M}=0
$$

where $\partial M$ is boundary of the manifold ${ }^{6}$. This system is gauge invariant if

$$
\left.\mathcal{B}^{[\alpha]} \partial_{\mu} \sigma\right|_{\partial M}=0
$$

This property simply means that space defined by (32) is invariant under the gauge transformations.

There are only two sets of gauge-invariant local boundary conditions for the Maxwell field ${ }^{7}$. Let us take one of them ${ }^{8}$ :

$$
\left.\alpha_{0}\right|_{\partial M}=0,\left.\quad \alpha_{\theta}\right|_{\partial M}=0,\left.\quad\left(\partial_{r}+\frac{1}{r}\right) \alpha_{r}\right|_{\partial M}=0,\left.\quad \sigma\right|_{\partial M}=0 .
$$

Obviously, if $\sigma$ satisfies Dirichlet boundary conditions, $\partial_{0} \sigma$ and $\partial_{\theta} \sigma$ also satisfy Dirichlet boundary conditions since $\partial_{0}$ and $\partial_{\theta}$ which act in tangential directions to the boundary. A bit more work is needed to show that the condition for $\alpha_{r}$ is also gauge invariant. Gauge transformation of the boundary condition (34) for $\alpha_{r}$ reads:

$$
\left(\partial_{r}+\frac{1}{r}\right) \partial_{r} \sigma=\left[-\Delta_{w} \sigma\right]+\left[-\frac{1}{r^{2}} \partial_{\theta}^{2}+2 \phi \phi^{*}\right] \sigma
$$

where we added and subtracted several terms such that the first bracket contains the operator (27) which defines eigenfrequencies in the ghost sector. We can expand $\sigma$ in a sum over eigenfrequencies: $\sigma=\sum_{k} \sigma_{k}$ so that $\Delta_{w} \sigma_{k}=\omega_{k}^{2} \sigma_{k}$ and each $\sigma_{k}$ satisfies Dirichlet boundary conditions as required by (34). Therefore,

$$
\left.\left[-\Delta_{w} \sigma\right]\right|_{\partial M}=-\left.\sum_{k} \omega_{k}^{2} \sigma_{k}\right|_{\partial M}=0
$$

\footnotetext{
${ }^{6}$ For Dirichlet boundary conditions the operator $\mathcal{B}$ is just the identity operator, so that $\left.\mathcal{B} \phi\right|_{\partial M}=0$ simply means $\left.\phi\right|_{\partial M}=0$. For Neumann boundary conditions $\mathcal{B}$ contains a normal derivative $\left(\partial_{r}\right.$ in our case). More complicated boundary operators will be introduced below.

${ }^{7}$ This point is discussed in the monographs [20,21], see also [22].

${ }^{8}$ Calculations for the other (dual) set of the boundary conditions go in a similar manner.
} 
This proves that the first term on the right hand side of (35) vanishes on the boundary. The second term there is also zero on the boundary since it does not contain normal derivatives acting on $\sigma$. We conclude, that the boundary conditions (34) are indeed gauge invariant.

Eigenfrequencies of $\sigma, \alpha_{0}$, and $w$ are defined by the same operator $\Delta_{w}$. Therefore, it is natural to impose on $w$ the same (Dirichlet) boundary conditions:

$$
\left.w\right|_{\partial M}=0
$$

Radial and angular components of $\alpha$ can be expressed through $\alpha_{+}$:

$$
\alpha_{r}=\Re\left(e^{-i \theta} \alpha_{+}\right), \quad \alpha_{\theta}=r \Im\left(e^{-i \theta} \alpha_{+}\right) .
$$

The operator $D_{F}^{\dagger} D_{F}$ acts on the bosonic fluctuations $\left(\varphi, i \alpha_{+} / \sqrt{2}\right)$ as well as on the fermionic components $V$ (cf. (24)). Hence, we impose the same boundary conditions on the lower component $V_{2}=\chi_{+}$as we have already defined for $i \alpha_{+} / \sqrt{2}$. Namely, ${ }^{9}$

$$
\left.\Re\left(e^{-i \theta} \chi_{+}\right)\right|_{\partial M}=0,\left.\quad\left(\partial_{r}+\frac{1}{r}\right) \Im\left(e^{-i \theta} \chi_{+}\right)\right|_{\partial M}=0 .
$$

To fix boundary conditions on the rest of the fields we shall use intertwining relations between $D_{F}^{\dagger} D_{F}$ and $D_{F} D_{F}^{\dagger}$. Let $U(\omega)$ and $V(\omega)$ be solutions of (23) with $\omega_{F}=\omega$. We can write formally:

$$
\begin{aligned}
& V(\omega)=\omega^{-2} D_{F}^{\dagger} U(\omega), \\
& U(\omega)=\omega^{-2} D_{F} V(\omega)
\end{aligned}
$$

for $\omega \neq 0$. We are looking for boundary conditions compatible with (40) and (41). Such boundary conditions will ensure that the operators $D_{F}^{\dagger} D_{F}$ and $D_{F} D_{F}^{\dagger}$ have coinciding non-zero eigenvalues.

Let us consider the first line in (41) which reads:

$$
U_{1}(\omega)=\omega^{-2}\left(D_{+} V_{1}(\omega)-\sqrt{2} e \phi V_{2}(\omega)\right) .
$$

Let us suppose that the boundary conditions for all components $U_{1}, U_{2}, V_{1}, V_{2}$ are mutually independent. This technical requirement will simplify the calculations below, but will not affect our main result. Let us take $V_{1}=0$ first. Then the first equation in (39) yields:

$$
\left.\Re\left(e^{-i \theta} \phi^{*} U_{1}\right)\right|_{\partial M}=\left.\Re\left(e^{-i \theta} \phi^{*} \psi_{+}\right)\right|_{\partial M}=0 .
$$

\footnotetext{
${ }^{9}$ Strictly speaking, eigenfrequencies of $\left(\varphi, i \alpha_{+} / \sqrt{2}\right)$ and $\left(V_{1}, V_{2}\right)$ are the same even if we identify respective boundary conditions up to a common constant phase factor. This freedom will be discussed in sec. 4.
} 


\begin{tabular}{|l|c|c|c|c|}
\hline Field & $\sigma$ & $\alpha$ & $w$ & $\varphi$ \\
\hline Equation & $(\underline{34})$ & $(\underline{34})$ & $(37)$ & $(\underline{47})$ \\
\hline
\end{tabular}

Table 1. Summary of the boundary conditions for ghosts and bosons.

\begin{tabular}{|l|c|c|c|c|}
\hline Field & $\psi_{+}=U_{1}$ & $\psi_{-}=V_{1}$ & $\chi_{+}=V_{2}$ & $\chi_{-}=U_{2}$ \\
\hline Equation & (43),$(48)$ & (45),$(46)$ & (39) & (45), (48) \\
\hline
\end{tabular}

Table 2. Summary of the boundary conditions for spinors.

Note, that we are not allowed to take normal derivative of (42) after we have put $V_{1}=0$ in order to get further conditions on $U_{1}$ since $\partial_{r}^{2} V_{1}$ is related to $V_{2}$ by the equations of motion, and, therefore, cannot be considered as an independent quantity on the boundary. Instead, we take the other component of (41):

$$
U_{2}(\omega)=\omega^{2}\left(\partial_{-} V_{2}-\sqrt{2} e \phi^{*} V_{1}\right)
$$

The boundary conditions (39) immediately give:

$$
\left.\Im\left(U_{2}\right)\right|_{\partial M}=0,\left.\quad \Im\left(\phi^{*} V_{1}\right)\right|_{\partial M}=0 .
$$

Next we return to (42) and put there $V_{2}=0$ to see that

$$
\left.\left(\partial_{r}-2\left(\partial_{r} \ln \phi^{*}\right)\right) \Re\left(\phi^{*} V_{1}\right)\right|_{\partial M}=0
$$

as a consequence of (43) and (45). Again, we identify the boundary conditions for $V_{1}$ with those for the first component of the boson doublet $\left(\varphi, i \alpha_{+} / \sqrt{2}\right)$ :

$$
\left.\Im\left(\phi^{*} \varphi\right)\right|_{\partial M}=0,\left.\quad\left(\partial_{r}-2\left(\partial_{r} \ln \phi^{*}\right)\right) \Re\left(\phi^{*} \varphi\right)\right|_{\partial M}=0 .
$$

Similarly, we use (40) to fix the boundary conditions for $U_{1}=\psi_{+}$and $U_{2}=\chi_{-}$:

$$
\left.\partial_{r} \Re\left(\chi_{-}\right)\right|_{\partial M}=0,\left.\quad\left(\partial_{r}+\frac{1}{r}\right) \Im\left(e^{-i \theta} \phi^{*} \psi_{+}\right)\right|_{\partial M}=0 .
$$

We have found a set of the boundary conditions which guarantees coincidence of non-zero eigenfrequencies for bosons and for fermions. We summarise the results of this section in Tables 1 and 2.

\section{Supersymmetry breaking at the boundary}

In the previous section we have constructed boundary conditions which support isospectrality of the operators acting in the bosonic and fermionic sectors. This suggests that certain degree of supersymmetry still remains in the problem even in the presence of boundaries. Due to the vortex, initial $N=2$ supersymmetry (8) is broken to the transformations with $\eta_{+}=0$. However, the other complex 
component $\eta_{-}$of the parameter $\eta$ remains unrestricted. In this section we show that in the presence of boundaries supersymmetry is broken to a real subgroup.

Let us consider the $\eta_{-}$transformation of $\alpha_{+}$:

$$
\delta \alpha_{+}=2 i \eta_{-}^{*} \chi_{+}
$$

From this equation we see that if $\eta_{-}$is an arbitrary complex parameter, it is not possible to impose different supersymmetric boundary conditions on real and imaginary parts of $\alpha_{+}$. For example, if $\Im\left(r e^{-i \theta} \alpha_{+}\right)=\alpha_{\theta}$ satisfies Dirichlet boundary conditions (as in our case), then because of (49) both real and imaginary parts of $\chi_{+}$should also satisfy Dirichlet boundary conditions. This, in turn, yields Dirichlet boundary conditions for $\Re\left(e^{-i \theta} \alpha_{+}\right)=\alpha_{r}$ contradicting gauge invariance of the boundary value problem.

However, if we require

$$
\Re\left(\eta_{-}\right)=0
$$

the boundary conditions obtained in the previous section become invariant under the supersymmetry transformations of the boson fields (i.e., boundary conditions are compatible with first there variations in (8)). This statement can be checked by direct and rather elementary calculations ${ }^{10}$. For example, compatibility of (49) is obvious since $\alpha_{+}$and $i \chi_{+}$satisfy the same boundary conditions.

Supertransformations (8) of the fermions are also compatible with our boundary conditions if $\Re\left(\eta_{-}\right)=0$. Proof of this statement (which is more involved than in the case of the bosons) is sketched in Appendix A.

One can change the residual supersymmetry by using the freedom mentioned above in the footnote 9. Since multiplication by a constant phase factor commutes with all operators and preserves normalisation of the eigenfunctions, one can replace the spinor field $\mathcal{F}=(U, V)$ by $\mathcal{F}_{\kappa}=e^{i \kappa} \mathcal{F}$ in the boundary conditions derived in sec. 3. However, this phase factor can be absorbed in a redefinition of the supersymmetry transformation parameter: $\eta \rightarrow \eta_{\kappa}=e^{i \kappa} \eta$. Then the supersymmetry transformations (8) remain the same in terms of $\mathcal{F}_{\kappa}, \eta_{\kappa}$. Supersymmetry of new transformed boundary condition would therefore require $\Re \eta_{\kappa-}=0$.

Let us stress, that the remaining supersymmetry is enough to achieve isospectrality of relevant operators in the bosonic and fermionic sectors. Of course, there is no guarantee that such cancellations will occur at higher loops as well. To understand the situation from the non-perturbative point of view one has to modify the Witten-Olive construction [24] accordingly.

\footnotetext{
${ }^{10}$ One has to take into account that complex conjugation of the Grassmann variables also changes order in their products. For example, $\left(\eta_{-}^{*} \chi_{-}\right)^{*}=\chi_{-}^{*} \eta_{-}$. Therefore, product of two real Grassmann variables is imaginary. Forgetting this property one would get $\Im\left(\eta_{-}\right)=0$ instead of (50) and a contradiction with superinvariance of the boundary conditions for fermions.
} 


\section{Quantum corrections to the mass of the vor- tex}

In the one-loop approximation the renormalised mass shift of the vortex consist of three terms:

$$
\Delta E^{\mathrm{ren}}=\Delta E(V+B)^{\mathrm{ren}}-\Delta E(B)^{\mathrm{ren}}+\Delta E^{\text {f.r. }}
$$

where the first term is the zero point energy in for the vortex in the spherical box, the second term is the energy associated with the boundaries of the box, and the third term is a contribution from finite renormalization of charges in the classical expression for the mass of the vortex.

In sec. 3] we have found such boundary conditions that all non-zero eigenfrequencies in the bosonic sector coincide with non-zero eigenfrequencies in the fermionic sector. Therefore, for a sufficiently large $s$ (cf. eq. (44)),

$$
\Delta E_{B}^{\mathrm{reg}}=\frac{1}{2} \sum_{\omega_{B} \neq 0} \omega_{B}^{1-2 s}=\frac{1}{2} \sum_{\omega_{F} \neq 0} \omega_{F}^{1-2 s}=\Delta E_{F}^{\mathrm{reg}} .
$$

If now we analytically continue (52) to $s=0$ we find that both divergent and finite parts of the vacuum energy for the vortex in the box are zero,

$$
\Delta E(V+B)^{\mathrm{ren}}=0 .
$$

This equation is, of course, valid for arbitrary radius $R$ of the box.

\subsection{Quantum energy of the boundaries}

Here we calculate the vacuum energy of the boundary of the box in the limit $R \rightarrow \infty$. First we have to show that between some characteristic radius $R_{1}$ (which is defined essentially by the size of the vortex) and $R$ the theory may be approximated by free massive fields.

As $r$ goes to infinity both profile functions of the vortex $f$ and $a$ go exponentially fast to their asymptotic values (16), (17). Therefore, near the boundary we can assume that $a$ and $f$ are constants and neglect their derivatives. Consequently, the operator $\Delta_{w}$ which defines the eigenfrequencies of $w, \alpha_{0}$ and of the ghosts $\sigma$ can be approximated by

$$
\tilde{\Delta}=-\partial_{j}^{2}+2 e^{2} v^{2}
$$

To understand what happens with the rest of the fields as $r \rightarrow \infty$ one has to analyse the operators (29) and (30). The Bogomolny equation (12) yields:

$$
\partial_{r} \phi=-\frac{i}{r} D_{\theta} \phi
$$


Consequently,

$$
\left(D_{-} \phi\right)=e^{-i \theta} 2 \partial_{r} \phi \rightarrow 0
$$

as $r \rightarrow \infty$. The same is true for $\left(D_{+} \phi^{*}\right)$, and both functions are approaching zero exponentially fast. This means that for large $r$ the off-diagonal terms in (29) can be neglected. The operators (29) and (30) contain the background vector potential (14) which does not vanish sufficiently fast at the infinity. This potential can be however transformed away by the following unitary change of variables for charged quantum fluctuations:

$$
\tilde{\varphi}=e^{i \beta(r) \theta} \varphi, \quad \tilde{\psi}=e^{i \beta(r) \theta} \psi,
$$

where the phase $\beta(r)$ is chosen in such a way that $\beta(r)=-n$ for $r>R_{1}$ and $\beta(r) \rightarrow 0$ insides the vortex. It is easy to see that in terms of new fields $\tilde{\varphi}$ and $\tilde{\psi}$ in the asymptotic region the eigenfrequencies are defined by the free operator (54) up to exponentially small terms.

One can easily show that not only the operators, but also the boundary conditions are identical in the bosonic and fermionic sectors up to exponentially small terms. Indeed, the fields $w, \alpha_{0}$ and $\sigma$ satisfy Dirichlet boundary conditions. Therefore, their contributions to the vacuum energy cancel also in the effective theory near the boundary. The fields $i \alpha_{+}, \chi_{+}$, and $\tilde{\psi}_{+}$satisfy:

$$
\begin{aligned}
& \left.\left(\partial_{r}+\frac{1}{r}\right) \Im\left(e^{-i \theta}\left(i \alpha_{+}, \chi_{+}, \tilde{\psi}_{+}\right)\right)\right|_{\partial M}=0, \\
& \left.\Re\left(e^{-i \theta}\left(i \alpha_{+}, \chi_{+}, \tilde{\psi}_{+}\right)\right)\right|_{\partial M}=0 .
\end{aligned}
$$

To derive the effective boundary conditions for $\tilde{\psi}_{+}$we have used that $e^{-i \beta(r) \theta} \phi^{*}$ goes exponentially fast to a constant when $r \rightarrow \infty$. Similarly we have:

$$
\begin{aligned}
& \left.\partial_{r} \Re\left(\left(\tilde{\varphi}, \tilde{\psi}_{-}, \chi_{-}\right)\right)\right|_{\partial M}=0, \\
& \left.\Im\left(\left(\tilde{\varphi}, \tilde{\psi}_{-}, \chi_{-}\right)\right)\right|_{\partial M}=0 .
\end{aligned}
$$

Taking into account a relative factor of $1 / 2$ in the contributions of spinors to the vacuum energy, we see that the total quantum energy associated with the effective field theory near the boundary is zero. This is true for arbitrary values of the regularization parameter, and, therefore,

$$
\Delta E(B)^{\text {ren }}=0 .
$$

\subsection{Finite renormalization}

As usual the renormalization is performed in the topologically trivial sector. We put $\phi=$ const. and calculate the effective potential. We shall not need other 
background fields. We use again the zeta function regularization as in (44). A real bosonic field with the mass $m$ contributes to the regularized effective potential

$$
W_{m}(s)=\frac{1}{2} \sum \omega(m)^{1-2 s}=\frac{1}{2} \zeta_{m}\left(s-\frac{1}{2}\right)
$$

where $\zeta_{m}$ is the zeta function for the operator $\Delta_{m}=-\partial_{j}^{2}+m^{2}$. It can be expressed through corresponding heat kernel:

$$
\zeta_{m}\left(s-\frac{1}{2}\right)=\Gamma\left(s-\frac{1}{2}\right)^{-1} \int d^{2} x \int_{0}^{\infty} d t t^{s-\frac{1}{2}-1} K(t, x) .
$$

The heat kernel reads

$$
K(t, x)=\left\langle x\left|e^{-t \Delta_{m}}\right| x\right\rangle=(4 \pi t)^{-1} e^{-m^{2} t} .
$$

The integral over $x$ in (62) is divergent due to the translational invariance of the background. Therefore, it is convenient to consider the density $\mathcal{W}: \int d^{2} x \mathcal{W}=$ $W$. The integration over $t$ can be easily performed. The subsequent analytic continuation to $s=0$ yields a finite result,

$$
\mathcal{W}_{m}=-\frac{m^{3}}{12 \pi}
$$

By collecting the contributions from all elementary excitations on this background we obtain:

$$
\mathcal{W}^{1-\text { loop }}=-\frac{e^{3}}{6 \pi}\left[\left(3|\phi|^{2}-v^{2}\right)^{3 / 2}-\left(2|\phi|^{2}\right)^{3 / 2}\right]
$$

Although the effective potential (65) is convergent in $2+1$ dimensions, there are finite renormalization effects which shift classical values of $e$ and $v$. To fix these shifts we consider

$$
\mathcal{W}^{\text {tot }}=\mathcal{W}^{\mathrm{cl}}(e+\hbar \delta e, v+\hbar \delta v)+\hbar \mathcal{W}^{1-\text { loop }}
$$

where we have re-inserted the $\hbar$ dependence. The first term on the right hand side is just the classical potential

$$
\mathcal{W}^{\mathrm{cl}}(e, v)=\frac{e^{2}}{2}\left(|\phi|^{2}-v^{2}\right)
$$

with shifted values of $e$ and $v$. We require that to the first order in $\hbar$ the potential $\mathcal{W}^{\text {tot }}$ has a minimum at $|\phi|=v$ ("no tadpole" condition). This condition yields

$$
\delta v=-\frac{e}{4 \sqrt{2} \pi} .
$$


To fix $\delta e$ one needs also another normalisation condition, but for our purposes (68) is already enough.

The shift (68) induces a shift in the vacuum energy:

$$
\Delta E^{\mathrm{f} . \mathrm{r} .}=\hbar(\delta v) \frac{d E^{\mathrm{cl}}}{d v}=-\frac{e v n \hbar}{\sqrt{2}} .
$$

Since other contributions (53) and (60) vanish,

$$
\Delta E^{\mathrm{ren}}=-\frac{e v n \hbar}{\sqrt{2}}
$$

This completes the calculation of the mass shift of the supersymmetric vortex.

\section{Conclusions}

In this paper we have re-calculated one-loop quantum corrections to the mass of the supersymmetric ANO vortex. We put the system into a box with a circular boundary and applied the zeta function regularization. We have demonstrated that boundaries violate a part of the supersymmetries, but the remaining invariances are enough to guarantee coincidence of the eigenfrequencies in the bosonic and fermionic sectors. Therefore, contributions from the bosons and the fermions to the vacuum energy cancel each other both in the full theory (vortex in a box) and in the effective theory near the boundary. Up to this point we agree with the previous works [7, 8, (though our conclusion is based on somewhat more reliable grounds). There is, however, a contribution (701) to the vacuum energy which comes from finite renormalization of the couplings in the classical mass of the vortex $^{11}$. Such contribution was neglected in the approach of $[7,8]^{12}$. To see what happens with the BPS bound one has to calculate also quantum corrections to the central charge.

Let us now give some comments on the vortex mass corrections in a pure bosonic theory. These comments are motivated by the discussion [26] on renormalization of the Casimir energy. In the supersymmetric case it was essential, that we the bosonic and fermionic contributions are cancelled mode-by-mode. In purely bosonic theory no such cancellation may appear and the vacuum energy will be, in general, divergent ${ }^{13}$. There are two types of the divergences which are given by volume or by boundary integrals. Normally, boundary divergences are the same in the full theory and in the effective theory defined near the boundary

\footnotetext{
${ }^{11}$ This situation is similar to the BPS black hole mass shift discussed in [25].

${ }^{12}$ It was pointed out to the present author by R.Wimmer that finite renormalizations will lead to a nonvanishing correction.

${ }^{13}$ In the zeta function regularization the one-loop divergences are defined by the heat kernel coefficients. For the (mixed) boundary conditions used in this work the heat kernel expansion can be found in [27].
} 
when $R \rightarrow \infty$. Therefore, $(\Delta E(V+B)-\Delta E(B))$ will contain volume divergences only which can be removed by some standard renormalization procedure. However, to define $\Delta E(V+B)$ or $\Delta E(B)$ separately one has to introduce new surface counterterms which are absent in the original model.

\section{Acknowledgements}

I am grateful to M. Bordag, A. Rebhan and especially to R. Wimmer for fruitful discussions. This work was supported in part by the DFG project BO 1112/12-1.

\section{A Supertransformation and boundary conditions}

In this Appendix we show how one can prove compatibility of our boundary conditions with the supersymmetry transformations of the spinor fields with pure imaginary $\eta_{-}$. Let us consider the supertransformation of $\chi_{-}$:

$$
\delta \chi_{-}=-\eta_{-}\left(\partial_{0} w+i \epsilon_{o j k} \partial^{j} \alpha^{k}-2 i e \Re\left(\phi^{*} \varphi\right)\right) .
$$

We are going to prove that $\delta \chi_{-}$satisfies the same boundary conditions as $\chi_{-}$if $\Re\left(\eta_{-}\right)=0$. The condition (45) on $U_{2}=\chi_{-}$can be checked easily:

$$
\left.\Im\left(\delta \chi_{-}\right)\right|_{\partial M} \sim-\left.\partial_{0} w\right|_{\partial M}=0
$$

where we have used the boundary condition (37). Let us now check the boundary condition (48):

$$
0=\left.\left.\partial_{r} \Re\left(\delta \chi_{-}\right)\right|_{\partial M} \sim \partial_{r}\left(-\epsilon_{o j k} \partial^{j} \alpha^{k}+2 e \Re\left(\phi^{*} \varphi\right)\right)\right|_{\partial M} .
$$

Consider the term on the right hand side of (73) which contains $\alpha$ :

$$
\begin{aligned}
-\partial_{r} \epsilon_{o j k} \partial^{j} & \left.\alpha^{k}\right|_{\partial M}=\left.\frac{1}{r}\left[\left(\partial_{r}-\frac{1}{r}\right) \partial_{r} \alpha_{\theta}+\frac{1}{r} \partial_{\theta} \alpha_{r}-\partial_{r} \partial_{\theta} \alpha_{r}\right]\right|_{\partial M} \\
& =\left.\frac{1}{r}\left[-(\Delta \alpha)_{\theta}-\left(\partial_{r}+\frac{1}{r}\right) \partial_{\theta} \alpha_{r}-\frac{1}{r^{2}} \partial_{\theta}^{2} \alpha_{\theta}\right]\right|_{\partial M} \\
& =-\left.\frac{1}{r}(\Delta \alpha)_{\theta}\right|_{\partial M}
\end{aligned}
$$

where we first re-expressed the left hand side through $\alpha_{r}$ and $\alpha_{\theta}$, then we used the vector Laplacian in the polar coordinates (cf., e.g., [23]):

$$
\begin{aligned}
& -(\Delta \alpha)_{r}=\left(\partial_{r}^{2}+\frac{1}{r} \partial_{r}+\frac{1}{r^{2}} \partial_{\theta}^{2}-\frac{1}{r^{2}}\right) \alpha_{r}-\frac{2}{r^{3}} \partial_{\theta} \alpha_{\theta} \\
& -(\Delta \alpha)_{\theta}=\left(\partial_{r}^{2}-\frac{1}{r} \partial_{r}+\frac{1}{r^{2}} \partial_{\theta}^{2}\right) \alpha_{\theta}+\frac{2}{r} \partial_{\theta} \alpha_{r} .
\end{aligned}
$$


Finally, to obtain the last line of (174) we made use of the boundary conditions (34). The equations of motion for $\alpha_{\theta}$ yield $^{14}$ :

$$
-\frac{1}{r}(\Delta \alpha)_{\theta}=-\frac{1}{r} \omega^{2} \alpha_{\theta}-2 e\left(\varphi^{*} \partial_{r} \phi+\varphi \partial_{r} \phi^{*}\right) .
$$

Now we collect all contributions to see

$$
\left.\left.\partial_{r} \Re\left(\delta \chi_{-}\right)\right|_{\partial M} \sim\left[-\frac{1}{r} \omega^{2} \alpha_{\theta}+e \Re\left(\phi^{*} \partial_{r} \varphi-\varphi \partial_{r} \phi^{*}\right)\right]\right|_{\partial M}=0
$$

due to (34) and (47).

Calculations for other components of the spinor fields can be done in a similar manner.

\section{References}

[1] A. A. Abrikosov, Sov. Phys. JETP 32, 1442 (1957); H. B. Nielsen and P. Olesen, Nucl. Phys. B 61, 45 (1973).

[2] A. Achucarro and T. Vachaspati, Phys. Rept. 327, 347 (2000) arXiv:hep-ph/9904229.

[3] A. I. Vainshtein and A. Yung, Nucl. Phys. B 614, 3 (2001) arXiv:hep-th/0012250.

[4] J. Edelstein, C. Nunez and F. Schaposnik, Phys. Lett. B 329, 39 (1994) arXiv:hep-th/9311055.

[5] Z. Hlousek and D. Spector, Nucl. Phys. B 370, 143 (1992); Nucl. Phys. B 397, 173 (1993).

[6] M. Bordag and I. Drozdov, Fermionic Vacuum Energy from a Nielsen-Olesen Vortex, arXiv:hep-th/0305002.

[7] J. R. Schmidt, Phys. Rev. D 46, 1839 (1992).

[8] B. H. Lee and H. Min, Phys. Rev. D 51, 4458 (1995) arXiv:hep-th/9409006.

[9] C. Imbimbo and S. Mukhi, Nucl. Phys. B 247, 471 (1984); Nucl. Phys. B 249, 143 (1985).

[10] C. Callias, Commun. Math. Phys. 62, 213 (1978). R. Bott and R. Seeley, Commun. Math. Phys. 62, 235 (1978).

\footnotetext{
${ }^{14}$ More precisely, the equation to follow is obtained by varying (21) with respect to $\alpha_{\theta}$ and then using the Bogomolny equation (12) for the background $\phi$.
} 
[11] E. J. Weinberg, Phys. Rev. D 19, 3008 (1979); Phys. Rev. D 20, 936 (1979).

[12] J. S. Dowker and R. Critchley, Phys. Rev. D 13 (1976) 3224; S. W. Hawking, Commun. Math. Phys. 55 (1977) 133. For a recent texbook on these methods, see E. Elizalde, Ten physical applications of spectral zeta-functions, (Springer, Berlin, 1995).

[13] A. Rebhan and P. van Nieuwenhuizen, Nucl. Phys. B 508, 449 (1997) arXiv:hep-th/9707163; H. Nastase, M. A. Stephanov, P. van Nieuwenhuizen and A. Rebhan, Nucl. Phys. B 542, 471 (1999) arXiv:hep-th/9802074; N. Graham and R. L. Jaffe, Nucl. Phys. B 544, 432 (1999) arXiv:hep-th/9808140; M. A. Shifman, A. I. Vainshtein and M. B. Voloshin, Phys. Rev. D 59, 045016 (1999) arXiv:hep-th/9810068.

[14] R. Wimmer, Quantization of supersymmetric solitons, arXiv:hep-th/0109119.

[15] M. Bordag, A. S. Goldhaber, P. van Nieuwenhuizen and D. Vassilevich, Phys. Rev. D 66, 125014 (2002) arXiv:hep-th/0203066.

[16] B. H. Lee, C. Lee and H. Min, Phys. Rev. D 45, 4588 (1992).

[17] E. B. Bogomolny, Sov. J. Nucl. Phys. 24, 449 (1976) [Yad. Fiz. 24, 861 (1976)].

[18] K. Fujikawa, B. W. Lee and A. I. Sanda, Phys. Rev. D 6, 2923 (1972); Y. P. Yao, Phys. Rev. D 7, 1647 (1973); L. Dolan and R. Jackiw, Phys. Rev. D 9, 2904 (1974).

[19] D. V. Vassilevich, Phys. Lett. B 421, 93 (1998) arXiv:hep-th/9709182.

[20] P. B. Gilkey, Invariance theory, the heat equation, and the Atiyah-Singer index theorem (CRC Press, Boca Raton, 1995).

[21] G. Esposito, A. Kamenshchik, and G. Pollifrone, Euclidean quantum gravity on manifolds with boundary (Kluwer, Dordrecht, 1997).

[22] D. V. Vassilevich, Phys. Rev. D 52, 999 (1995) arXiv:gr-qc/9411036.

[23] D. V. Vassilevich, J. Math. Phys. 36, 3174 (1995) arXiv:gr-qc/9404052.

[24] E. Witten and D. I. Olive, Phys. Lett. B 78, 97 (1978).

[25] S. J. Rey, Nucl. Phys. B 508, 569 (1997) arXiv:hep-th/9610157. 
[26] N. Graham, R. L. Jaffe, V. Khemani, M. Quandt, M. Scandurra and H. Weigel, Nucl. Phys. B 645, 49 (2002) arXiv:hep-th/0207120; K. A. Milton, Calculating Casimir energies in renormalizable quantum field theory, arXiv:hep-th/0210081, S. A. Fulling, Systematics of the Relationship between Vacuum Energy Calculations and Heat Kernel Coefficients, arXiv:quant-ph/0302117.

[27] T. P. Branson, P. B. Gilkey, K. Kirsten and D. V. Vassilevich, Nucl. Phys. B 563, 603 (1999) arXiv:hep-th/9906144. 